\title{
Hierarchical Selection Theory and Sex Ratios I. General Solutions for Structured Populations
}

\author{
STEVEn A. Frank \\ Division of Biological Sciences, University of Michigan, \\ Ann Arbor, Michigan 48109-1048
}

Received July 6, 1984

\begin{abstract}
Models of sex-ratio evolution in structured populations are derived with G. R. Price's covariance form for the hierarchical analysis of natural selection (1970, Nature 227, 520-521). Previous work on competition among related males for mates (local mate competition), competition among related females for a limiting resource (local resource competition), inbreeding, group selection, and asymmetry of genetic inheritance between males and females, are subsumed under a general formulation for sex-ratio biases in structured populations. I found that the evolutionarily stable strategy sex ratio (males: females) for diploids is $1-\rho_{m}: 1-\rho_{f}$, where $\rho_{m}$ is the regression coefficient of relatedness of the controlling genotypes on males competing for mates, $\rho_{f}$ is the regression of controlling genotypes on females that compete for a fixed, limiting resource, and there is no inbreeding. For inbreeding and no competition among females, the evolutionarily stable strategy is $1-\rho_{m}: 1+\rho_{m f}$, where $\rho_{m f}$ is the regression of controlling genotypes on females' mates. $\quad c 1986$ Academic Press, Inc.
\end{abstract}

Many interesting behaviors reflect a tension between the selfish pursuits of some individual or entity within a local group, and the extent to which the ultimate success of that individual also depends on the vigor of its local group. To name just a few of the most popular puzzles, there are the (almost) sterile castes of social insects, in which workers rarely produce offspring, but perhaps gain by the greater success of their colony, which contains a high proportion of identical genes (Hamilton, 1964, 1972). There is the kin group, where the principle of self-sacrifice for a relative depending on the level of relatedness (Hamilton, 1964) has been accepted as one of the guiding principles of behavioral ecology. Meiotic and gametic drive are further examples - the increased success of part of the genome that possibly reduces the overall success of the genome (e.g., Haldane, 1932; Hamilton, 1967; Alexander and Borgia, 1978; Eberhard, 1980; Cosmides and Tooby, 1981). The most recent slogan capturing this idea is "selfish DNA with self-restraint": genetic elements that can replicate and spread within the genome; but the ultimate success of the elements (relative 
rate of propagation) also depends on the success of the genome, and so prudent production is favored (Doolittle et al., 1984). The list is endless and itself fascinating, the natural outgrowth of the hierarchical organization of life (e.g., Lewontin, 1970; Hamilton, 1975, 1978; Alexander and Borgia, 1978; Wilson, 1980; Frank, 1983a).

A great deal of effort has been devoted to generating and arguing about terms and styles of explanation for hierarchical phenomena (e.g., Charlesworth and Toro, 1982; Borgia, 1982; Wildish, 1982; Colwell, 1982; Harvey et al., 1985; Nunney, 1985; and reviews of Doolittle, 1982; Wilson, 1983; Grafen, 1984). The arguments have often suffered for lack of a suitable formal description that is both general and captures in an intuitive manner the crux of the problem. Formal treatments of particular subjects have usually been mathematically ingenious and complex, but are impenetrable for most, and not well suited for generalization. There is, however, a simple and powerful method for analyzing natural selection in hierarchically organized settings (Price, 1970, 1972a), which has only recently received much attention (see next section for references). Here I illustrate this general method for hierarchical selection by unifying a segment of sex-ratio theory which has been very controversial, and which has proved difficult to analyze formally.

Sex ratios have received a great deal of empirical and theoretical attention [see reviews by Charnov (1982) and Frank (1983a)]. Empirically, sex ratios can be measured with much greater precision than most other traits that are so clearly associated with fitness. Thus, sex-ratio predictions are testable. There are two related reasons for the theoretical excitement. First, the fitness accruing to the producer of a particular sex ratio is frequency dependent. When males are rare, making males yields a high fitness, and when males are common, producing them is a strategy with low fitness (Fisher, 1930). At some sex ratio the reproductive returns on energy invested in sons equals the reproductive returns on energy invested in daughters, and the producer of this allocation enjoys the greatest fitness (reviewed by Charnov, 1982). Therefore, when an individual allocates its resources into some proportion of male and female reproductive functions (e.g., staminate versus pistillate flowers, sons versus daughters, etc.), the best allocation in terms of fitness depends on what other members of the population are doing. Charnov's (1982) synthesis of the theory and empirical evidence regarding sex allocation demonstrates the richness of this subject.

It is well known that under frequency dependent selection phenotypes that maximize individual fitness are often suboptimal for the subpopulation or population (reviewed by Wright, 1969). This leads to the second aspect of excitement about sex-ratio theory. The sex ratio that yields the greatest fitness within a local group or subpopulation is different from the sex ratio 
that maximizes the growth of the group. When the groups differ in their genetic composition, the fitness of an individual within the population will depend on both its success within the group, and the group's success within the population (Hamilton, 1975, 1979; Colwell, 1981; Wilson and Colwell, 1981). And in much the same way, different subsets of the genome may increase their rate of propagation at a sex ratio different from other subsets of the genome. For example, matrilineally inherited genes are passed only to daughters, and so favor a female-biased sex ratio (Shaw, 1958; Hamilton, 1967); while autosomal genes are passed equally to sons and daughters, and favor equal allocation of resources into each sex (Fisher, 1930; Shaw and Mohler, 1953; Charnov, 1982). These sorts of hierarchical conflicts over the sex ratio, among different parts of the genome within an individual, individuals within a group, and groups within the population were revicwed in a previous paper (Frank, 1983a). In the present study hierarchical selection theory is formally applied to the simplest sorts of sexratio problems in which hierarchical conflicts exist. New results are derived, and both new and old work are unified within a general framework for sex ratios in structured populations.

\section{Hierarchical Selection Theory}

Several workers discovered independently that an elegant formulation of natural selection can be derived by treating fitness as a quantitative phenotypic character, so that the intensity of section depends on the covariance between fitness and additive genotypic value (Robertson, 1966, 1968; Li, 1967a, 1967b, 1976; Price, 1970, 1972a; Crow and Kimura 1970). The simplest version of the model is $A q=\operatorname{Cov}(w, Q) / \bar{w}$, where $q$ is a population allele frequency, $w$ is the fitness of a particular genotype, $\bar{w}$ is population fitness, and $Q$ is the additive genotypic value representing the allele frequencies within each individual (e.g., if $q$ is the frequency of $a$, and $p$ of $A, p+q=1$, then $A A$ is assigned the genotypic value $0, A a$ the value 0.5 , and $a a$ the value 1$)$. This simple covariance selection formula allowed $\mathrm{Li}$ (1967a, 1976) and Price (1972b) to clarify Fisher's $(1930,1941,1958)$ Fundamental Theorem of Natural Selection. Seger (1981) used this model of selection to synthesize previous work on the appropriate coefficients of relatedness for kin selection theory, developing a new measure that subsumes previously used coefficients. Uyenoyama (1984a) also used the covariance approach to develop a genetic representation of the cost of meiosis.

Price $(1970,1972 a)$ developed a more general version of the covariance formulation that allows the components of natural selection to be partitioned in a hierarchical fashion. [For further discussion of the method, 
see Hamilton (1975), Arnold and Fistrup (1982), and Wade (1985).] Hamilton (1975), Wade (1980), Uyenoyama (1984b), and Ohta (1983) have given some applications of this hierarchical model to simple group selection and altruism problems. Three features of Price's formulation make it simultaneously simple, exceedingly general, and intuitively meaningful in its biological applications.

(i) The equation treats two successive levels of selection contributing to a single overall process.

$$
\bar{w} \Delta q=\operatorname{Cov}\left(w_{d}, q_{d}\right)+E\left(w_{d} \Delta q_{d}\right) .
$$

The levels analyzed may be freely chosen. Thus $q$ may refer to the frequency of any sort of particle or unit. Typically, however, the allelic, transposon, chromosomal, or individual level will be represented by $q$. $d$ can refer to any grouping of units represented by $q$, such as alleles grouped within an individual, individuals grouped within a subpopulation, etc. A $d-$ type group contains a unit (e.g., allele) frequency $q_{d}$. The population fitness represented by $\bar{w}$ may refer to any higher grouping of units above, $d$, e.g., a traditional biological population, a symbiotic association, or the biosphere. The covariance term quantifies the contribution of among-group selection, while the expectation term describes within-group selection, thus formally separating the two processes.

(ii) The equation can be used to expand itself, and thus fitness can be partitioned into any number of hierarchical levels. Replacing the covariance term by the product of the regression and the variance, and using the equation to expand the last term, one obtains

$$
\begin{aligned}
\bar{w} \Delta q= & R\left(w_{d}, q_{d}\right) V\left(q_{d}\right)+\sum_{d} \alpha_{d}\left[R_{d}\left(w_{d i}, q_{d i}\right) V_{d}\left(q_{d i}\right)\right. \\
& \left.+E_{d}\left(w_{d i} \Delta q_{d i}\right)\right]
\end{aligned}
$$

where $\alpha_{d}$ is the frequency of $d$-type groups.

(iii) Each term of the equation has a natural biological interpretation. As an example, let $q$ represent the frequency of a particular allele, $d i$ an individual within a $d$-type group, and $d$ a group of individuals within the population. Then $\Delta q_{d i}$ represents the change in allele frequency among the set of successful gametes within the dith individual, $R_{d}\left(w_{d i}, q_{d i}\right)$ is the slope of the fitnesses with respect to additive genotypic values of $d i$ individuals within $d$-type groups (i.e., strength of within-group selection), and $R\left(w_{d}, q_{d}\right)$ is the slope of group fitnesses within the population with respect to genotype frequencies of the groups (i.e., strength of among-group selection). $V\left(q_{d}\right)$ is the among-group variance, and $V_{d}\left(q_{d i}\right)$ are the within- 
group variances. These variance terms quantify the relative importance of the among-group and within-group selection coefficients, and highlight the role that among-unit variances play at all levels of selection.

\section{SOLUTIONS FOR FIVE SCENARIOS}

There are three goals of this section. First, the generality of hierarchicat selection theory is illustrated by deriving sex-ratio predictions for the common population structure scenarios that have been discussed in the literature, thus unifying this segment of sex-ratio theory. Second, solutions for this set of scenarios allow the various causal mechanisms underlying the predicted sex-ratio biases to be evaluated. Third, the results suggest very general, qualitative sex-ratio trends that are predicted under the conditions of each scenario. These scenarios are quite artificial, and are not meant to be an accurate description of any real organism. A priori predictions for particular organisms must be derived from the organisms' specific natural history details; an example of specific predictions and a relevant data set for thig wasps are presented in a second paper (Frank, 1985).

\section{Local Mate Competition}

Fisher (1930) asserted that the sex ratio will evolve so that the energy invested in males equals the energy invested in females. The essence of Fisher's reasoning was that a member of the rarer sex would, on average, leave more offspring than a member of the commoner sex. Hence, the argument goes, more grandchildren accrue to a producer of the rarer sex, and eveutually there follows the population-wide equal investment in the sexes.

Hamilton (1967) noted two latent assumptions in Fisher's theory. First, the genes that control the sex ratio must be inherited in a manner uncorrelated with sex (Shaw and Mohler, 1953; Shaw, 1958). There is much evidence that suggests that a strong association exists between cytoplasmic (maternal) or sex-linked inheritance patterns and highly skewed investment ratios in the sexes (e.g., Bateson and Gairdner, 1921; Rhoades, 1933; Howard, 1942; Zimmering et al., 1970; Werren et al., 1981; Skinner, 1982; Frank, 1983a; Laughnan and Gabay-Laughnan, 1983). When these inheritance patterns occur, a parent is asymmetrically related (AR) to male and female offspring (see also Hamilton, 1972; Trivers and Hare, 1976; Alexander and Sherman, 1977; Charnov, 1978; Uyenoyama and Bengtsson, 1981, 1982). The second assumption Hamilton (1967) noted is that there must be genetically random competition for mates among males (i.e., there is no local mate competition, LMC). In his 1967 
paper Hamilton gave analytic results for two simple scenarios. Later, Hamilton (1979) presented a general solution that simultaneously addresses AR and LMC, and includes the 1967 results as special cases (see also Uyenoyama and Bengtsson, 1982). Combining AR and LMC is particularly useful, since the organisms most commonly studied for LMC are haplodiploid (Charnov, 1982), which is genetically equivalent to sex linkage and male heterogamety (XX females and $\mathrm{XO}$ males), thus violating the symmetric inheritance assumption when the mother is inbred (see below).

In the remainder of this section, (i) Hamilton's (1979) general result for combining AR and LMC is reviewed and the previously reported prediction for haplodiploidy is derived as an example, (ii) the assumptions underlying this general result are presented, (iii) a proof of this result is given by using hierarchical selection theory, and (iv) the assumptions and difficulties of the method are discussed. With the details of this scenario explained, the next four scenarios will require only new or altered assumptions and a sketch of the derivations.

\section{General Result for $A R$ and $L M C$}

Hamilton's (1979) model for the evolutionarily stable strategy (ESS of Maynard Smith and Price, 1973) sex ratio (males/total) when the symmetric inheritance and random mate competition assumptions are violated can be written as

$$
r^{*}=(1 / 2) R P_{d t}
$$

where (1/2) is Fisher's (1930) equal investment result, $R$ is Hamilton's (1979) coefficient of inheritance asymmetry between the sexes, and $P_{d t}$ is Wright's (1969) index of panmixia. $R=2 B_{p s} /\left(B_{p s}+B_{p d}\right)$, where $B_{p s}$ and $B_{p d}$ are Hamilton's (1972) "complete" regression coefficients of relatedness at the sex-ratio locus for the parent that controls the sex ratio to son and daughter, respectively, or for the sex of the sibling that controls the brood sex ratio to brother and sister, respectively (Trivers and Hare, 1976; Uyenoyama and Bengtsson, 1982). These coefficients are proportional to the inclusive fitness benefit (or genetic valuation) to a parent of an act directed toward a son or daughter, and are equivalent to the weighted coefficient $G^{\prime}$ in the review on coefficients of relatedness by Pamilo and Crozier (1982). A genetic element on a diploid autosome in a parent is equally likely to be in a son or a daughter, so inclusive fitness benefits are equal for acts directed at sons and daughters, and $R=1$. For elements on a $Y$ chromosome in heterogametic males (or for a patrilineally inherited element), $B_{f s}=1$ and $B_{f d}=0$, so $R=2$. For elements on a $Y$ (or $W$ ) chromosome in heterogametic females (or a matrilineally inherited 
element), $B_{m s}=0$ and $B_{m d}=1$, so $R=0$. The values of $B$ for elements on an $X$ chromosome can be obtained from Table I. For example, if females are $\mathrm{XX}$, males $\mathrm{XY}$ or $\mathrm{XO}$ (as in haplodiploids), and the sex ratio is maternally controlled, then $B_{m s}=1 / 2$ and $B_{m d}=(1+3 F) /(2+2 F)$, and $R=$ $(1+F) /(1+2 F)$, where $F$ is Wright's fixation index, here measuring the extent to which individuals are inbred (Wright, 1969). Formally, $P_{d t}$ is the expected within-deme variance in additive genotypic values divided by the population variance, and here characterizes the population structure. By construction of the model, $P_{d t}$ measures both the genotypic correlation among competing males (LMC) and the genotypic correlation between mates (see the later section on the interpretation of biased sex ratios). With knowledge of the type of genetic control, with $F$, the index of inbredness known for calculating $R$, and with $P_{d t}$ known to set the level of LMC and inbreeding, a specific prediction for $r^{*}$ can be derived.

Throughout the paper, I will use the term inbredness to mean the correlation $F$ between alleles within an individual (or sometimes individual's alleles plus mate's alleles) that controls the sex-ratio phenotype. The term inbreeding will be used to describe the genotypic correlation $\left(1-P_{d t}\right)$ between mates among the set of individuals that are the expression of the sex-ratio phenotype. In the usual case, the mother controls the sex-ratio phenotype, and inbredness refers to the correlation among her alleles plus here mate's alleles (mated composite); and inbreeding refers to the genotypic correlation between the mothers of mated pairs (see below). This may seem rather tortuous, but the distinction is important for clarifying the causal mechanisms underlying biased sex ratios, an issue which has been very muddled in the past. I thank R. K. Colwell for suggesting these two terms to help clarify the distinction betwecn the two ways in which forms of inbreeding affect sex ratios.

Before giving the proof for (3), I derive as an example the result for maternal control in haplodiploids. To obtain values for $F$ and $P_{d t}$,

TABLE I

"Complete" or "Life-for-Life" Regression Coefficients of Relatedness of Parents to Offspring for Sex-Linked Loci (from Hamilton, 1972; see also Uyenoyama and Bengtsson, 1982, Table IV).

\begin{tabular}{|c|c|c|c|}
\hline & & \multicolumn{2}{|c|}{ Offspring } \\
\hline & & $\mathrm{XY}$ or $\mathrm{XO}$ & $x x$ \\
\hline Controlling parent & $\begin{array}{c}\mathrm{XY} \text { or } \mathrm{XO} \\
\mathrm{XX}\end{array}$ & $\begin{array}{c}F \\
1 / 2\end{array}$ & $\begin{array}{c}1+F \\
(1+3 F) /(2+2 F)\end{array}$ \\
\hline
\end{tabular}


assumptions are needed about the population structure. The usual scenario for studying LMC consists of eight assumptions. (i) The population is divided into an infinite number of demes, each colonized by a constant number of females, $N$. (ii) Females mate randomly with members of the deme in which they were born, and store the sperm. (iii) Within their deme, males compete randomly with respect to genotype against other males. (iv) The deme disintegrates after one generation and the mated females disperse and colonize new demes. (v) Each female invests a constant amount of energy in offspring, $K$. (vi) The cost of producing a female equals the cost of producing a male, hence sex ratio equals sex investment ratio. This assumption is probably not necessary for most conditions (MacNair, 1978; Uyenoyama and Bengtsson, 1982), however, all my studies were based on number of offspring, rather than amount of energy allocated to each sex. (vii) Male's only investment in offspring is sperm, and each male can potentially fertilize many females. In addition to these seven assumptions, it was also usually assumed that the already mated females settle into new demes each generation in a random fashion with respect to genotype. Under these assumptions, the inbredness of an individual is $F=y /(4-3 y)$, where $y$ is the amount of sib mating in each generation ( $\mathrm{Li}, 1976)$. In this scenario the probability of sib mating is $1 / N$, hence $F=1 /(4 N-3)$. Using Table I for haplodiploids, $R=(4 N-2) /(4 N-1)$. The additive genotypic variance within a deme is a sampling variance, $s^{2}$, for a random sample of size $N$. The expected value of $s^{2}$ is $[(N-1) / N] V_{l}$, where $V_{l}$ is the population variance (see the final section for the interpretation of variance terms). Hence $P_{d t}$ is $(N-1) / N$, and

$$
r^{*}=(1 / 2)[(4 N-2) /(4 N-1)][(N-1) / N]
$$

as reported by Hamilton (1979), Taylor and Bulmer (1980), and Uyenoyama and Bengtsson (1982). Uyenoyama and Bengtsson (1982) showed, with different techniques and under slightly different model assumptions, that (4) is actually unstable to all other sex ratios. Biological interpretations of this result remain unclear: for example, a complex evolutionary dynamic may ensue, or the result may be stable on a small neighborhood surrounding (4). Equation (4) has gained qualitative empirical support (Wilson and Colwell, 1981; Werren, 1983; other studies reviewed in Charnov, 1982), but quantitative discrepancies between this prediction and observations have been noted (Hamilton, 1979; Frank, 1983a, 1983b). Many of the assumptions underlying (4) are unrealistic. Assumptions and predictions that are more realistic are presented in a second paper (Frank, 1985). 
Assumptions and Proof.

The proof of (3) is modified from a set of unpublished notes kindly made available to me by W. D. Hamilton. The first seven assumptions are the same as listed above for the haplodiploid case. The eighth assumption above, that females settle randomly, is not needed. Any pattern of settling is allowed. In addition to these seven assumptions: (viii) The sex ratio, $r$, is controlled by a single locus that may be autosomal, sex-linked, or cytoplasmic. (ix) If the locus is not autosomal, $R$ will differ for maternal versus paternal control of the sex ratio. Only one parent will control the sex ratio, and $R$ will be for that parent (usually the mother). (x) Individual females that are already mated and store sperm are treated as phenotypic sex-ratio units, consisting of their own genotypes plus that of the stored sperm. (xi) The only type of within-sex competition is local competition for mates among males. Food and other resources are not limiting for either males or females.

The argument in this section will be cast as a phenotypic argument; in the next section the terms will be redefined to show how this argument relates to a fully genetical one. Since the goal here is to find an ESS phenotype (i.e., sex ratio), the phenotypic argument is easier to follow intuitively. The fully genetical explanation serves to show that while some formal mathematical difficulties exist, they do not seem to harm the qualitative interpretations.

Equation (3) will be obtained by finding the sex ratio $r^{*}$ that cannot be bettered by a type producing a different sex ratio, either higher or lower: $r^{*}$ is therefore an equilibrium sex ratio in a sense of being evolutionarily stable against invasion once established (i.e., an ESS, Maynard Smith and Price, 1973). This solution is achieved by searching for the sex ratio produced by a type such that, for any other type producing a sex ratio different from $r^{*}$, with frequency represented by $q, \bar{w} \Delta q$ of (2) is always less than zero.

To begin, we need expressions for the terms in (2). Let there be two sexratio types ("type" is the phenotype produced by the genes of a female plus the genes of her mate): $a$ which produces sex ratio $r_{a}$, with frequency represented by $p$, and $b$ which produces sex ratio $r_{b}$, with frequency represented by $q$. Let the subscript $i$ represent individuals within demes colonized by $N$ females, and demes be indexed by the subscript $d$. Demes within the population are described by the number of $b$ types they contain, $d=0,1,2, \ldots, N$, with associated probabilities $\alpha_{d} . p_{d}$ and $q_{d}$ are the frequencies of the $a$ and $b$ types, respectively, within a $d$-type deme $\left(q_{d}=d / N\right)$, and $r_{d}=p_{d} r_{a}+q_{d} r_{b}=r_{a}-\Omega q_{d}$, where $\Omega=r_{a}-r_{b}$ is the difference between the sex ratios of the two types. In a $d$-type deme, the expected number of inseminations per son is $\left(1-r_{d}\right) / r_{d}$, the number of females divided by the number of males in the deme. This is the Fisherian frequency dependent 
valuation of sons. Thus for an $a$ type, the expected number of inseminations by sons is proportional to $r_{a}\left[\left(1-r_{d}\right) / r_{d}\right]$, the number of daughters is proportional to $1-r_{a}$, and the genetic valuation of a son is $B_{p s}$, and of a daughter, $B_{p d}$. So

$$
\left.w_{d i}(a)=r_{a} \mathrm{~L}\left(1-r_{d}\right) / r_{d}\right] B_{p s}+\left(1-r_{a}\right) B_{p d},
$$

likewise for $w_{d i}(b)$, replacing $a$ with $b$. Measures similar to $w_{d i}$, the genetic representation in future generations, have been used by Shaw and Mohler (1953) and MacArthur (1965); see also "effective fitness" of Uyenoyama and Feldman (1981) and "representational fitness" of Uyenoyama and Bengtsson (1982). Since there are two sex-ratio types, $R_{d}\left(w_{d i}, q_{d i}\right)=$ $w_{d i}(b)-w_{d i}(a)$, or, setting $B_{p s}+B_{p d}=B$,

$$
R_{d}\left(w_{d i}, q_{d i}\right)=-\Omega\left(B_{p s} / r_{d}-B\right)
$$

And,

$$
\begin{aligned}
w_{d} & =p_{d} w_{d i}(a)+q_{d} w_{d i}(b)=\left(1-r_{d}\right) B_{p s}+\left(1-r_{d}\right) B_{p d} \\
& =\left(1-r_{d}\right) B=\left(1-r_{a}+\Omega q_{d}\right) B .
\end{aligned}
$$

The gradient of $w_{d}$ on $q_{d}$ can be taken directly from (7):

$$
R\left(w_{d}, q_{d}\right)-\Omega B
$$

By assumption $(\mathrm{x})$, individuals are considered indivisible types, therefore $\Delta q_{d i}=0$ (see below for justification). Substituting into (2),

$$
\bar{w} \Delta q=\Omega B V\left(q_{d}\right)-\Omega \sum \alpha_{d} V_{d}\left(q_{d i}\right)\left(B_{p s} / r_{d}-B\right)
$$

where $\sum$ is the summation over $d$ unless otherwise noted. Using a standard method for locating an ESS [described by Maynard Smith (1982) and Uyenoyama and Bengtsson (1982)],

$$
\left.\frac{\partial \bar{w} \Delta q}{\partial r_{b}}\right|_{r_{a}=r_{b}=r^{*}}=-B V\left(q_{d}\right)+\left(B_{p s} / r^{*}-B\right) \sum \alpha_{d} V_{d}\left(q_{d i}\right)=0 .
$$

The derivative with respect to $r_{b}$ locates the best sex ratio for the $b$ types (greatest value of $\bar{w} \Delta q$ ) assuming $r_{a}$ is fixed. At $r_{a}=r_{b}$ there is no selection, so $\bar{w} \Delta q=0$; thus evaluating the derivative at this point defines $r_{a}=r^{*}$ such that $\bar{w} \Delta q \leqslant 0$ for all $r_{b}$ on $[0,1]$, since the second derivative is everywhere negative on [0,1], and when $r_{a}=r^{*}, \bar{w} \Delta q$ can be shown to be negative for $r_{b}$ close to $r_{a}$. (Restricting $r_{b}$ to be close to $r_{a}$ is required since $R$ depends on the intensity of selection, see below.) Since $\sum \alpha_{d} V_{d}\left(q_{d i}\right)$ is the expected 
variance within a deme, $V_{w d}$, and $V\left(q_{d}\right)$ is the among-group variance, it follows that $V_{w d}+V\left(q_{d}\right)=V_{t}$, where $V_{t}$ is the total variance. Solving,

$$
r^{*}=\left(B_{p s} / B\right)\left(V_{w d} / V_{t}\right)=(1 / 2) R P_{d t}
$$

\section{Discussion of Assumptions and Method}

There are four types of assumptions that need to be mentioned: (i) stochastic effects, (ii) conditional behavior, (iii) asymmetry between the sexes, and (iv) the assumption of sex-ratio phenotypes without specifying the underlying genetic basis.

(i) Stochastic effects. The model assumes, unrealistically, such things as (a) no variance in male success within a deme, (b) no variance in number of offspring per female, (c) all demes formed by exactly $N$ foundresses, and (d) all females of a particular sex-ratio phenotype producing exactly the same sex ratio. Assumptions (b) and (c) will be relaxed and treated as stochastic in a model presented in the second paper (Frank, 1985). The general method can probably also be extended to treat (a) and (d) as stochastic. When (a) does not hold, the ESS appears to become more female biased (Wilson and Colwell, 1981); when (b) is violated, the effective deme size is reduced, again causing the ESS to become more female biased (Charnov, 1982; Frank, 1983b). If deme size varies, then the expected value of $P_{d t}$ is likely to be affected (Frank, 1983b), and the effect of a nonzero variance in the sex ratio around the ESS has not been explored.

(ii) Conditional behavior. The model assumes that an individual produces a genetically fixed sex ratio. It is well known that certain organisms, such as the haplodiploid Hymenoptera, can adjust their sex ratio according to local conditions (see Charnov, 1982). Situations in which individuals are allowed to vary their sex ratios in response to certain local conditions have been discussed in the literature (Trivers and Willard, 1973; Werren and Charnov, 1978; Charnov et al., 1981; Clutton-Brock et al., 1981; Werren, 1980, 1983), and a few such models are presented in Frank (1985). For example, the proportion of males produced by fig wasps is positively correlated with deme size (Frank, 1983a, 1983b).

(iii) Asymmetry between the sexes. The models in this paper assume that males do not invest anything in offspring except sperm, and that a single male can potentially fertilize many females. When males do divide up some resource among their progeny, the success of each progeny decreases with increasing number of inseminations. A model reflecting male investment could easily be contructed by adjusting $w_{d i}$ in Eq. (5). 
(iv) Genetical basis of phenotypes. Finding an ESS phenotype is equivalent to asking if there is a particular phenotype from some specified set that, when near fixation in the population, is such that no other rare phenotype enjoys a greater fitness. The argument is constructed by studying the direction of evolution between all possible pairs of phenotypes. This was the method used in the previous section, but much still needs to be explained. Selection is most easily thought of as occurring among phenotypes, and so it was simplest to set up the expressions for fitness, $w_{d i}$, as depending on the frequency of phenotypes, as in all derivations of this paper. But in sexual populations phenotypes are, of course, not inherited whole, so phenotypic fitness is an approximate sort of reasoning.

A rigorous population genetics model for partial sib mating (and thus also some competition among brothers, or LMC) and for all types of genetic control is given by Uyenoyama and Bengtsson (1982). They obtained the general result $r=(1 / 2) R(1-k)$, when there is no dominance and $k$ is the proportion of sib mating. They discuss conditions for an ESS to exist, and distinguish rates of approach to the ESS for various scenarios. Their results are equivalent to Eq. (3) under the above LMC scenario. The relationship between $k$ and $P_{d t}$ is discussed in the final section of this paper.

Phenotypic success, as used in the above derivation, must be translated into genetic success. In the derivation, it was assumed that only two sexratio phenotypes exist. One phenotype was designated (algebraically) to always be the more successful type, denoted the " $a$ type." Now let us first consider the simplest case: autosomal control of the sex ratio. A gene in a parent is equally likely to be in a son or a daughter, so the number of copies of a gene passed on to the grandprogeny generation is directly proportional to the number of grandprogeny, and no special weighting needs to be given to a grandchild through a son versus a daughter. $w_{d i}$ in (5) with $B_{p d}=B_{p s}=1$ is proportional to the number of grandprogeny for a particular phenotype, and so is a measure of the rate of propagation of any autosomal gene carried by that phenotype, ignoring meiotic drive, gametic selection, and sampling effects (drift). The notation needs to be redefined. Assume that the $a$ type is homozygous, $A A$ (with a genotypic value of 0 ), at the single locus affecting the sex ratio, with any number of alternate alleles. Any individual that is not $A A$ at this locus is designated a " $b$ type" ( $A B$ with a genotypic value of 0.5 , and $B B$ a value of 1 ), and the fitness of the $b$ types, $w_{d i}(b)$ in the models, is the average fitness over all non- $A A$ genotypes, where "types" here refers to the genotypes of the parent that control the sex ratio. $p$ now refers to the frequency of allele $A$, and $q$ to the frequency of all other alleles at the sex-ratio locus. $\Delta q_{d i}$, the change in gene frequency within the set of successful gametes of individual $d i$, can be taken as zero, since meiotic drive, gametic selection, and drift are assumed 
negligible. With these assumptions the allele $A$ must be increasing in frequency in each generation, for any sort of dominance relationship and any number of alternate alleles, and for any original frequency of $A$, assuming $p$ is not so small that $A A$ homozygotes are rare. The only problem is that $P_{d t}$ is actually set out to measure both the genotypic correlation between mates and the correlation among males competing for mates (see final section of this paper), and so $P_{d t}$ depends on both the genotype frequencies and the difference between $r_{a}$ and the sex ratios of the $b$ types, $r_{b}$. Thus deriving a constant-valued ESS depends on further assumptions to fix $P_{d t}$ : that $q$ be rare and that $r_{b}$ (for all $b$ types) be close to $r_{a}$. These assumptions are implicit for the ESSs reported in this paper. [It is often easy to show that the equilibrium is stable against any value of $r_{b}$ by setting $r_{a}=r^{*}$, and checking the sign of $\bar{w} \Delta q$ for all $r_{b}$. Hamilton (unpublished) has shown this for (11) when $R=1$; sec also Colwell (1981), Wilson and Colwell (1981), and Uyenoyama and Bengtsson (1979, 1981, 1982).]

For nonautosomal control, the number of grandprogeny through a son needs to be weighted by the the probability that a son will transmit a replica of a parental gene relative to the probability that a daughter will transmit a replica of a parental gene. Such a weighting is the regression coefficient of relatedness developed by Hamilton $(1964,1970,1972)$ and later analyzed in greater detail by several authors (reviewed by Seger, 1981; Uyenoyama and Feldman, 1982). Uyenoyama and Bengtsson (1979, 1981, 1982) prove that this method of weighting sons and daughters is correct for sex-ratio problems, subject to the difficulty of properly specifying the coefficients of relatedness, which depend on the frequency of genotypes, the intensity of selection, and the form of dominance.

In deriving (4), the ESS sex ratio for haplodiploidy under maternal control, the coefficient of relatedness was obtained by using the values of $F$ and $P_{d t}$ expected for a neutral locus $\left(r_{b}\right.$ very close to $r_{a}$ ). Since (4) was also obtained by studying the recurrence relations for all possible genotypes and random mating within demes (Taylor and Bulmer, 1980), using $F$ and $P_{d t}$ for a neutral locus appear valid under certain circumstances. Uyenoyama and Bengtsson (1982) reported that for every model they studied, the coefficient of relatedness expected under no selection is the only possible candidate for an ESS, when the common allele is either completely dominant, completely recessive, or there is no dominance. Perhaps because an ESS type must do better than all possible types, and the second derivative of $\bar{w} \Delta q$ with respect to $r_{b}$ is a negative constant (i.e., $\bar{w} \Delta q$ as a function of $r_{b}$ is a smooth curve with a single peak at $\left.r_{a}=r_{b}=r^{*}\right)$, the only situation in which the $b$ type poses a serious threat to the $a$ type is when $r_{b}$ is very close to $r_{a}$, and hence the rate of selection is vanishingly slow. (For general discussions of the relationship between phenotypic and genetic models, see Maynard Smith, 1982; Grafen, 1984.) 


\section{Local Resource Competition}

Clark (1978) noted that in some primates males disperse from their natal groups, while females remain at home and compete among themselves for a limited resource. Under these conditions, which she called "local resource competition" (LRC), she suggested that a male-biased sex ratio is favored. Bulmer and Taylor (1980a) and Taylor (1981) have also concluded that different dispersal distances of the two sexes will potentially cause more sib competition within one sex, and that the sex ratio will be biased in favor of the sex with the smaller degree of sib competition.

The LRC scenario is the same as the LMC scenario with the following modifications: (a) male offspring disperse to compete and mate randomly within the population, and (b) female offspring within a deme divide among themselves a fixed, limited resource. With these modifications, the value per female in a $d$-type deme is proportional to $1 /\left(1-r_{d}\right)$, since females split up a fixed resource; and the average value per female in the population is $\sum\left[\alpha_{d} /\left(1-r_{d}\right)\right]$. The expected number of inseminations per male is the number of females divided by the number of males, or $(1-r) / r$; where $r$ is the population sex ratio, $\sum \alpha_{d} r_{d}$. For an $a$ type, the investment in males is $r_{a}$, and in females, $1-r_{a}$. So

$w_{d i}(a)=r_{a}[(1-r) / r]\left[\sum \alpha_{d} /\left(1-r_{d}\right)\right] B_{p s}+\left[\left(1-r_{d}\right) /\left(1-r_{d}\right)\right] B_{p d}$.

Expressions necessary to substitute into (2) are derived as in the LMC scenario, and the same method is used to solve for the ESS:

$$
r^{*}=B_{p s} /\left(B_{p s}+B_{p d} P_{d t}\right)
$$

where $P_{d t}$ now measures the genetic differentiation among groups of females, and there is competition for a fixed, limited resource within the group. With random settling and autosomal control of the sex ratio, $r^{*}=$ $N /(2 N-1)$, which agrees with Hamilton's unpublished result cited by Charnov $(1982$, p. 75$)$ that was derived by methods similar to those in Hamilton (1967) (Hamilton, personal communication).

\section{The Haystack Model}

Bulmer and Taylor (1980b) and Wilson and Colwell (1981) have studied two scenarios similar to the LMC scenario. In the first, $g$ generations occur in each deme, with only local mating, until the $g$ th generation, when mated females disperse to colonize new demes. In the second, $g-1$ generations occur in each deme, then in the $g$ th generation both sexes disperse before mating, and mate randomly within the population before settling into new 
demes to begin the cycle again. The algebra presented below for analyzing the first scenario is tedious, but the derivation points to previously hidden processes underlying the predicted sex ratios, and is also a good illustration of the heuristics involved in applying the Price equation to ESS analyses.

A generalization of (2) is necessary in order to analyze this scenario. Let superscripts refer to generation: $q_{d}^{(j)}$ is the frequency of $b$ types among parents of the $j$ th progeny generation, and $w_{d i}^{(j)}$ is the fitness in a $d$-type deme for an individual parent of the $j$ th generation, and $w_{d}^{(g)}$ is the cumulative fitness of a $d$-type deme over $g$ generations. Generalizing (2) so that $\bar{w} \Delta q$ refers to one set of $g$ generations within demes, and up to and including the founding of new demes to begin the next set of $g$ generations,

$$
\begin{aligned}
\bar{w} \Delta q= & R\left(w_{d}^{(g)}, q_{d}^{(1)}\right) V\left(q_{d}^{(1)}\right) \\
& +\sum \alpha_{d}\left[R_{d}\left(w_{d i}^{(g)}, q_{d i}^{(1)}\right) V_{d}\left(q_{d i}^{(1)}\right)\right],
\end{aligned}
$$

assuming $\Delta q_{d i}=0$, as in the LMC scenario. For simplicity I assume autosomal control of the sex ratio, so one can take $B_{p s}=B_{p d}=1$, and $B=2$, since $B_{p s}$ and $B_{p d}$ are the relative weights of sons and daughters. Reasoning as in the LMC scenario, Eq. (7), $w_{d}^{(j)}=\left(1-r_{d}^{(j)}\right) B=2\left(1-r_{d}^{(j)}\right)$, so

$$
w_{d}^{(g)}=\prod w_{d}^{(j)}=2^{g} \prod\left(1-r_{d}^{(j)}\right)
$$

where $\Pi$ is the product over $j=1,2, \ldots, g$, unless otherwise noted. Similarly,

$$
\begin{aligned}
w_{d i}^{(g)}(a) & =\prod w_{d i}^{(j)}(a)=\prod\left[r_{a}\left(1-r_{d}^{(j)}\right) / r_{d}^{(j)}-\left(1-r_{a}\right)\right] \\
& =\prod\left(r_{a} / r_{d d}^{(j)}+1-2 r_{a}\right)
\end{aligned}
$$

and,

$$
R_{d}\left(w_{d i}^{(g)}, q_{d i}^{(1)}\right)=w_{d i}^{(g)}(b)-w_{d i}^{(g)}(a)=C_{2 d} .
$$

Noting from Eq. (1) and (2) that

$$
\begin{aligned}
R\left(w_{d}^{(g)}, q_{d}^{(1)}\right) V\left(q_{d}^{(1)}\right) & =\operatorname{Cov}\left(w_{d}^{(g)}, q_{d}^{(1)}\right) \\
& =\sum \alpha_{d} w_{d}^{(g)}\left(q_{d}^{(1)}-q^{(1)}\right) \\
& =2^{g} \sum \alpha_{d}\left(q_{d}^{(1)}-q^{(1)}\right) \prod\left(1-r_{d}^{(j)}\right)=C_{1},
\end{aligned}
$$


we now have

$$
\begin{gathered}
\bar{w} \Delta q=C_{1}+\sum \alpha_{d} C_{2 d} V_{d}\left(q_{d i}^{(1)}\right)=C_{3} \\
\frac{\partial C_{1}}{\partial r_{b}}=2^{g} \sum_{d} \alpha_{d}\left(q_{d}^{(1)}-q^{(1)}\right) \sum_{j}\left[\Omega\left(q_{d}^{(j)} / r_{b}\right)-q_{d}^{(j)}\right) \prod\left(1-r_{d}^{(k)}\right) \\
\frac{\partial C_{2 d}}{\partial r_{b}}=\sum_{j} \frac{r_{d}^{(j)}+r_{b}\left[\Omega\left(q_{d}^{(j)} / r_{b}\right)-q_{d}^{(j)}\right]}{\left(r_{d}^{(j)}\right)^{2}}-2 \prod\left(r_{b} / r_{d}^{(k)}+1-2 r_{b}\right) \\
+\sum_{j} \frac{r_{a}\left[q_{d}^{(j)}-\Omega\left(q_{d}^{(j)} / r_{b}\right)\right]}{\left(r_{d}^{(j)}\right)^{2}} \prod\left(r_{a} / r_{d}^{(k)}+1-2 r_{a}\right)
\end{gathered}
$$

where $\Pi$ is the product over $k=1,2, \ldots, g$, with $k \neq j$. At $r_{a}=r_{b}=r^{*}$, $\Omega=0$, and $q_{d}^{(j)}=q_{d}^{(1)}$ for all $j$, and it follows that

$$
\begin{aligned}
\left.\frac{\partial \bar{w} \Delta q}{\partial r_{b}}\right|_{r_{b}=r_{b}=r^{*}}= & -g(2)^{g}\left(1-r^{*}\right)^{g-1} \sum_{d} \alpha_{d}\left(q_{d}^{(1)}-q^{(1)}\right) q_{d}^{(1)} \\
& +g(2)^{g-1}\left(1-r^{*}\right)^{g-1} \sum_{d} \alpha_{d}\left(1 / r^{*}-2\right) V_{d}\left(q_{d i}^{(1)}\right)=0 \\
= & -2 V\left(q_{d}^{(1)}\right)+\left(1 / r^{*}-2\right) V_{w d}^{(1)}=0 .
\end{aligned}
$$

Solving,

$$
r^{*}=(1 / 2) P_{d t}
$$

This result agrees with the central LMC result in the first scenario, with $R=1$, and is independent of the number of generations $g$ in the haystack. This disagrees slightly with the numerical analysis results of Bulmer and Taylor (1980b), who found that the ESS sex ratio decreases slightly as $g$ increases. At the present time the meaning of this discrepancy is not clear. Perhaps my method has ignored sampling effects within the groups in each generation, which would increase the among-group variance and decrease $P_{d t}$ in each generation (see also Grafen, 1984).

A heuristic argument may help clarify the general approach, and particularly this last result for the haystack model. First, let us return to the one-generation LMC scenario with diploid autosomal control of the sex ratio, and $N=2$ females settling randomly (with respect to genotype) into each deme. Let the number of offspring per female be 4 . This is simply a device to insure that we locate the ESS, which we know to be $r^{*}=$ $(1 / 2)[(N-1) / N]=1 / 4$. Table II is a matrix showing the expected number of grandchildren for female I when she produces the sex ratio given by the 
TABLE II

Expected Number of Grandchildren for Female I in a Deme

Founded by Two Already-Mated Females

\begin{tabular}{ccccccc}
\hline & \multicolumn{5}{c}{ Female II } \\
\hline & $0 / 4$ & $1 / 4$ & $2 / 4$ & $3 / 4$ & $4 / 4$ \\
\hline \multirow{4}{*}{ Female I } & $0 / 4$ & & 16 & 16 & 16 & 16 \\
& $1 / 4$ & 40 & $24^{*}$ & 18.6 & 16 & 14.4 \\
& $2 / 4$ & 32 & 21.3 & 16 & 14.8 & 10.6 \\
& $3 / 4$ & 24 & 16 & 9.2 & 8 & 5.7 \\
& $4 / 4$ & 16 & 9.6 & 5.3 & 2.3 & \\
\hline
\end{tabular}

Note. The number of grandchildren by female I, calculated by the method described in the text, depends on the sex ratio of each female, where rows are female I's sex ratio (male/total) and columns are female II's sex ratio. The total number of grandchildren for both females I and II depends on the total number of daughters, accounting for the asymmetry in the matrix. Expected numbers of grandchildren for female II are obtained by transposing the matrix. [A similar table can be found in Frank (1983a) and Maynard Smith (1983); see also Hamilton (1967).] Presenting the number of grandchildren is a measure of relative fitness within the total population. If each entry is divided by average fitness within the group $[=(i, j+j, i) / 2]$, then the new entries are the relative fitnesses within groups. Such a transformation shows that $2 / 4$ is the only stable sex ratio, which is consistent with Fisher's (1930) theory, as stressed by Colwell (1981) and Wilson and Colwell (1981). In the final analysis, however, all that matters is relative fitness with respect to the entire population, and the within-group fitnesses only serve to sharpen one's intuition about the process.

rows and female II produces the sex ratio given by the columns. The sex ratio of $1 / 4$ is the only stable equilibrium - it is the greatest number of grandchildren that female I can obtain when female II produces $1 / 4$, and it is the greatest number that female II can obtain when female I produces $1 / 4$. The expected number of grandchildren is $4 \cdot$ (number of daughters) + $4 \cdot$ (number of sons) $\cdot\left[\left(1-r_{d}\right) / r_{d}\right]$, where $\left(1-r_{d}\right) / r_{d}$ is the expected number of inseminations per male.

In the first generation of a haystack model with random settling of females, assume female I produces $r^{*}$ and female II produces $r \neq r^{*}$. Then female 1 will have a greater genetic representation within the population (although not within the group, Table II) among the mated females of the second generation (daughters plus stored sperm), and there will be effectively less than two females colonizing the next generation. If female 1 , producing $r^{*}$, continues to hold an advantage in each generation (in terms of number of descendants) over female II, producing $r \neq r^{*}$, then $r^{*}$ is an ESS. If female II's descendants do better in some future generation, then the number of descendants for I and II will converge (assuming smooth, 
continuous behavior of frequencies), and the effective number of foundresses again approaches $N=2$, where $r^{*}$ will again prevail.

This discussion actually describes a haploid model, and ignores some realistic complexities. These include the Mendelian mode of inheritance, the tendency of groups to diverge because of drift - thereby increasing $P_{d t}$, the tendency of selection to cause divergence among groups, and so on.

A common criticism of the haystack model is that it allows unrestricted, exponential growth of demes for $g$ generations between each dispersal episode (e.g., Wilson and Colwell, 1981). Clearly such exponential growth is unrealistic for anything but small clutch sizes and few generations. When a limit is placed on the size a deme can attain, the effect of group selection is reduced, and the equilibrium sex ratio tends back toward the Fisherian $1 / 2$.

To develop a model with a limit on the number of individuals a deme can support, I first present some notation. $N$ is the number of foundresses colonizing a deme; $k$ is the number of offspring per female in each generation; $K$ is the carrying capacity per deme, here defined as number of individuals the deme can hold without any density dependent reduction of fitness; the $j$ th generation is the $j$ th parental generation, where the 1 st parental generation is the original founding females, and $r_{d}^{(j)}$ is the sex ratio produced by the $j$ th parental generation in a $d$-type deme; $T_{d j}$ is the number of individuals in deme $d$ in the $j$ th parental generation (which is the $(j-1)$ st progeny generation) under unrestricted growth, $T_{d j}=N k^{j-1} \Pi\left(1-r_{d}^{(n)}\right)$, where $\Pi$ is the product over $n=1,2, \ldots, j-2 ; N_{d j}$ is the number of individuals in a $d$-type deme in the $j$ th parental generation, $h_{d j}$ is the discount to the fitness of the $j$ th parental generation due to density dependent factors

$$
\begin{gathered}
h_{d j}=\left(K / N_{d j}\right)^{\phi_{d j}} \quad \phi_{d j}=0 \quad \text { if } \quad N_{d j}<K \\
=1 \quad \text { if } \quad N_{d j} \geqslant K \\
N_{d j}=T_{d j}=w_{d}^{(j-1)} T_{d, j-1} \quad j=1,2, \ldots, m_{d}+1 \\
N_{d j}=w_{d}^{(j-1)}\left(N_{d, j-1}\right)\left(h_{d, j-1}\right)=w_{d}^{(j-1)}\left(N_{d, j-1}\right)\left(K / N_{d, j-1}\right) \\
=w_{d}^{(j-1)} K \quad j=m_{d}+2, m_{d}+3, \ldots, g+1
\end{gathered}
$$

where $m_{d}+1$ is the first parental generation that is greater than $K$ in size. With this notation, generation refers to parental generation, so the $(g+1)$ st generation is the $g$ th generation produced within the stack, and is the dispersing generation that becomes the first generation of the next episode of breeding. In this model mating occurs before dispersal. Since the 
$\left(m_{d}+1\right)$ st generation is the first parental generation that is greater than or equal to $K$, it is also the $m_{d}$ th progeny generation. Keeping in mind the notation for generations, we can write expressions for the fitness discount $h_{d j}$ :

$$
\begin{aligned}
h_{d j} & =1 \quad N_{d j}=T_{d j}<K & & j=1,2, \ldots, m_{d} \\
& =K / N_{d j} \quad N_{d j}=T_{d j} \geqslant K & & j=m_{d}+1 \\
& =K / N_{d j}=K / w_{d}^{(j-1)} K=1 / w_{d}^{(j-1)} & & j=m_{d}+2, m_{d}+3, \ldots, g+1 .
\end{aligned}
$$

Before proceeding to the derivation, the usage of "deme" (or "group" or "patch" in the literature) needs to be clarified (this issue is discussed further in the final section of the paper). It has been shown (Colwell, 1981; Wilson and Colwell, 1981) that differential productivity of groups is a necessary condition for female-biased sex ratios in LMC and haystack scenarios. There are, however, many different ways in which group structure affects sex ratio. Consider the standard LMC scenario with $g=1$. By the notation developed here, the first progeny generation is the second, or $(g+1)$ st parental generation. The $(g+1)$ st generation is always the generation that disperses and includes foundresses of the next cycle. The fitness discount $h_{d, g+1}$ can be treated in one of three general ways, which correspond to three different ways in which group structure affects sex ratios. (i) Set $h_{d, g+1}=1$; that is, the fitness of the dispersing $(g+1)$ st parental females does not depend on competition for resources before dispersal within the natal deme. In this case, when $g=1$, a limit on the number of progeny by the first (founding) parental generation has no effect on the predicted sex ratio. (ii) $h_{d, g+1}$ is calculated as in (24). If $g=1$, there would not be any effect on the sex ratio due to the discount (here the usual LMC result, (3), applies), since growth of the deme and discount to females is not dependent on the sex ratio produced, because both sons and daughters use local resources. If $g>1$, the discount may affect the sex ratio by reducing the effect of group selection. There is no advantage to either individuals within the group, or to the group within the population, of surpassing a group size of $K$. Thus, depending on the parameters, within-group components will be weighted more heavily. (iii) Females of the dispersing $(g+1)$ st generation split up a fixed resource. Thus, the sum of the fitnesses over all dispersing females of a deme is a constant, $K_{f}$, independent of the number of females $N_{d, g+1}\left(1-r_{d}^{(g+1)}\right)$, and $h_{d, g+1}=K_{f} / N_{d, g+1}\left(1-r_{d}^{(g+1)}\right)$. This is an LRC scenario, and is a way in which differential productivity of groups can be suppressed for $g=1$ (Colwell, 1981; Wilson and Colwell, 1981). As Colwell (1981) and Wilson and Colwell (1981) point out for suppression of differential productivity for $g=1, r^{*}=1 / 2$ (see next scenario). 
The general form of (2) for the three cases is

$$
\begin{aligned}
\bar{w} \Delta q= & R\left(w_{d}^{(\mu)} h_{d \mu}, q_{d}^{(1)}\right) V\left(q_{d}^{(1)}\right) \\
& +\sum_{d} \alpha_{d} R_{d}\left(w_{d i}^{(\mu)} h_{d \mu}, q_{d i}^{(1)}\right) V_{d}\left(q_{d i}^{(1)}\right)
\end{aligned}
$$

where $w_{d}^{(\mu)} h_{d u}$ is the cummulative realized fitness of a $d$-type deme after $\mu$ generations; and for case (i) $\mu=g$, for case (ii) $\mu=g+1$ and $h_{d, g+1}$ as in (24), and for case (iii) $\mu=g+1$ with $h_{d, g+1}=K_{f} / N_{d, g+1}\left(1-r_{d}^{(g+1)}\right)$. Here I analyze one case (i) for autosomal control of the sex ratio. The expressions for the regressions are as in the first haystack model, with appropriate weightings for $h_{d j}$ :

$$
\begin{aligned}
& \bar{w} \Delta q=\sum_{d} \alpha_{d} 2^{g}\left(q_{d}^{(1)}-q^{(1)}\right) \prod_{j} h_{d j} \prod_{j}\left(1-r_{d}^{(j)}\right) \\
& +\sum_{d} \alpha_{d} V_{d}\left(q_{d i}^{(1)}\right)\left\{\prod _ { j } h _ { d j } \left[\prod_{j}\left(r_{b} / r_{d}^{(j)}+1-2 r_{b}\right)\right.\right. \\
& \left.\left.-\prod_{j}\left(r_{a} / r_{d}^{(j)}+1-2 r_{a}\right)\right]\right\} \\
& =\sum_{d} \alpha_{d} \prod_{j} h_{d j}\left(C_{3}\right) \\
& \left.\frac{\partial \bar{w} \Delta q}{\partial r_{b}}\right|_{r_{a}=r_{b}=r^{*}}=\left.\prod_{j} h_{j} \frac{\partial\left(C_{3}\right)}{\partial r_{b}}\right|_{r_{a}=r_{b}}+\left.\frac{\partial \prod h_{d j}}{\partial r_{b}}\right|_{r_{a}=r_{b}}\left(C_{3}\right)_{r_{a}=r_{b}}=0
\end{aligned}
$$

where $h_{i j}=h_{d j}$ at $r_{a}=r_{b}$ for all $d$, and $\left(C_{3}\right)$ is the right-hand side of (19). So

$$
\begin{aligned}
\left.\frac{\partial \bar{w} \Delta q}{\partial r_{b}}\right|_{r_{a}=r_{b}}= & \prod_{j} h_{j}\left[-2 g V\left(q_{d}^{(1)}\right)+g\left(1 / r^{*}-2\right) V_{w d}^{(1)}\right] \\
& +2\left(1-r^{*}\right) \sum_{d} \alpha_{d}\left(q_{d}^{(1)}-q^{(1)}\right)\left(\partial \prod_{j} h_{d j} / \partial r_{b}\right)=0 \\
\left.\frac{\partial \prod_{j} h_{d j}}{\partial r_{b}}\right|_{r_{a}=r_{b}=r^{*}}= & \sum_{j}\left(\partial h_{d j} / \partial r_{b}\right) \prod_{k} h_{k} \\
= & \sum_{j} h_{j}\left(\partial \ln h_{d j} / \partial r_{b}\right) \prod_{k} h_{\cdot k} \\
= & \prod_{j} h_{j} \sum_{d}\left(\partial \ln h_{d j} / \partial r_{b}\right)
\end{aligned}
$$


where products over $k$ are from 1 to $g$, with $k \neq j$. Continuing,

$$
\begin{aligned}
\ln h_{d j}=\phi_{d j} \ln \left(K / N_{d j}\right) & =0 & & T_{d j}=N_{d j}<K \\
& =\ln \left(K / N_{d j}\right) & & T_{d j}>K .
\end{aligned}
$$

From (24), $K / N_{d j}=K / T_{d j}$ for $j=m_{d}+1$, and $K / N_{d j}=1 / w_{d}^{(j-1)}$ for $j>m_{d}+1$. Now confine $r_{b}$ to the neighborhood of $r_{a}$ and search only for a local equilibrium. Then, $w_{d}^{(j-1)}$ will change very little over $j \geqslant m_{d}+1$, especially if a few generations have gone by before the $\left(m_{d}+1\right)$ st, so set $w_{d}^{(j-1)}=w_{d}^{(+)}$for all $j \geqslant m_{d}+1$, and

$$
\begin{array}{rlrl}
\ln h_{d j} & =0 & j & =1,2, \ldots, m_{d} \\
& =-\ln w_{d}^{(+)} & j & =m_{d}+1, m_{d}+2, \ldots, g .
\end{array}
$$

For $j \geqslant m_{d}+1$

$$
\frac{\partial \ln h_{d j}}{\partial r_{b}}=-\left(1 / w_{d}^{(+)}\right)\left[2\left(\Omega q_{d}^{(j)} / r_{b}\right)-q_{d}^{(j)}\right] \quad j=m_{d}+1, \ldots, g .
$$

Setting $k=2$ and reparameterizing $K$ accordingly, at $\Omega=0$ (29) reduces to $q_{d}^{(1)} /\left(1-r^{*}\right)$; and, with $\Omega=0, m_{d}=m$ for all $d$. So

$$
\left.\sum_{j} \frac{\partial \ln h_{d j}}{\partial r_{b}}\right|_{r_{a}=r_{b}=r^{*}}=(g-m)\left(q_{d}^{(1)}\right) /\left(1-r^{*}\right)
$$

$$
\begin{aligned}
\left.\frac{\partial \bar{w} \Delta q}{\partial r_{b}}\right|_{r_{a}=r_{b}=r^{*}}= & -2 g V\left(q_{d}^{(1)}\right)+g\left(1 / r^{*}-2\right) V_{w d}^{(1)} \\
& +2\left(1 \quad r^{*}\right) \sum_{d} \alpha_{d}\left(q_{d}^{(1)}-q^{(1)}\right)(g-m)\left(q_{d}^{(1)}\right) /\left(1-r^{*}\right) \\
= & -2 g V\left(q_{d}^{(1)}+g\left(1 / r^{*}-2\right) V_{w d}^{(1)}+2(g-m) V\left(q_{d}^{(1)}\right)=0\right. \\
r^{*}= & \frac{(1 / 2) P_{d t}}{m / g+(1-m / g) P_{d t}} .
\end{aligned}
$$

Recall that $m+1$ is the parental generation that first exceeds the density independent carrying capacity, $K$, so $m=0$ implies the carrying capacity has been achieved by the foundresses in the first parental generation, and $r^{*}=1 / 2$, as one would expect. When $m=g$, the density dependent interactions are not a factor, and $r^{*}=(1 / 2) P_{d t}$, agreeing with (22), and when $0<m<g$, then $1 / 2\left(P_{d t}\right)<r^{*}<1 / 2$. It may seem than when $m_{d}<g$ for all $d$, that the ESS should be $1 / 2$. But by forcing the same sex ratio in each generation for a particular sex-ratio type, the ESS is some weighting of the advantage accruing to producers of many colonizing females in the final, 
dispersing generation, and the within-group selection that increases in relative strength as $g / m$ increases. Thus, under the condition $m_{d}<g$ for all $d$, if a type can produce one sex ratio for $g-1$ generations, and a different one in the $g$ th generation, then it seems likely that the ESS for the first $g-1$ generations is to produce $r^{*}=1 / 2$, and in the $g$ th parental generation is to produce $r^{*}=(1 / 2) P_{d t}$, where $P_{d t}$ is the variance among the $g$ th parental generation (which at equilibrium should be the same as in the first generation, since both will produce the ESS of $1 / 2$ during the first $g-1$ generations).

\section{$L M C, L R C$, and Dispersal}

This scenario and the next are designed to expose the mechanisms underlying biased sex ratios. Here the basic scenario is again the one generation per deme LMC model. Among the progeny generation of $N$ females (defining a deme), a proportion $m$ of the males compete for mates with males from their natal deme, and $1-m$ disperse and compete randomly within the population. A proportion $f$ of the females compete for a limiting resource with females from their natal deme, and $1-f$ disperse and compete with females for a limiting resource randomly within the population. By construction of the model, mating is assumed random for all individuals (i.e., no inbreeding), for both dispersers and those that remain and compete with individuals of the same sex from their natal demes. Differential dispersal of the two sexes has also been explored by Bulmer and Taylor (1980a) and Taylor (1981).

A situation in nature that may parallel this scenario is the social insects, where colonies of certain species specialize either in the production of male or female reproductives (Herbers, 1979; Pamilo, 1982; Ward, 1983). In this case there can be no sib mating, and a proportion of the reproductives (either male or female) will compete with their sibs for either mates or quality nest sites, while a proportion will disperse. Further, selection will favor a proportion of the reproductives to disperse in order to avoid competition with their sibs (Hamilton and May, 1977). These ideas about social insects arise from comments by R. D. Alexander following a seminar by R. L. Trivers in 1985 .

For a dispersing male, the expected number of inseminations is $(1-r) / r$, and the value per insemination (i.e., the value per female) is proportional to $1 /(1-r)$. For a male in the natal deme, the expected number of inseminations is $(1-r) / r_{d}$, and the value per insemination is $1 /(1-r)$. The value of a dispersing female is $1 /(1-r)$, and for a female that competes in her natal deme, $1 /\left(1-r_{d}\right)$, so the adaptive function is: 


$$
\begin{aligned}
w_{d i}(a)= & (1-m) r_{a}[(1-r) / r][1 /(1-r)]+m r_{a}\left[(1-r) / r_{d}\right][1 /(1-r)] \\
& +(1-f)\left(1-r_{a}\right)[1 /(1-r)]+f\left(1-r_{a}\right)\left[1 /\left(1-r_{d}\right)\right] \\
= & (1-m)\left(r_{a} / r\right)+m\left(r_{a} / r_{d}\right)+(1-f) \\
& \times\left[\left(1-r_{a}\right) /(1-r)\right]+f\left[\left(1-r_{a}\right) /\left(1-r_{d}\right)\right]
\end{aligned}
$$

Regression coefficients and the derivation are obtained as in the first LMC scenario

$$
r^{*}=\frac{1-m\left(1-P_{d t}\right)}{2-(m+f)\left(1-P_{d t}\right)}=1-m\left(1-P_{d t}\right): 1-f\left(1-P_{d t}\right)
$$

where $X: Y=X /(X+Y)$. For random settling of foundresses, $P_{d t}=(N-1) / N$, so

$$
r^{*}=(1-m / N):(1-f / N)=N-m: N-f .
$$

When $m=1$ and $f=0, r^{*}=N-1: N=(N-1) /(2 N-1)$, as reported by Werren (1983). The ratio form is (genetic valuation of sons): (genetic valuation of daughters) and is extremely useful for exploring the mechanisms underlying sex-ratio biases. This technique will be exploited in the discussion, but first one last scenario is needed.

\section{LMC with Dispersal}

This scenario is the one-generation LMC model with a few modifications. There is no LRC (competition among females), a proportion $m$ of the males stay within their natal deme and compete locally for mates; $1-m$ of the males disperse as a group to mate randomly within the population, but compete only among themselves for mates. Females mate within their natal demes, and mate with a deme member with probability $m$ and a nonmember with probability $1-m$. With these assumptions,

$$
w_{d i}(a)=m r_{a}\left[\left(1-r_{d}\right) / r_{d}\right]+(1-m) r_{a}\left[(1-r) / r_{d}\right]+1-r_{a} .
$$

By the usual methods

$$
r^{*}=\frac{P_{d t}}{(m+1)-(m-1) P_{d t}}=P_{d t}: 1+m\left(1-P_{d t}\right) .
$$

When $m=1, r^{*}=(1 / 2) P_{d t}$, which is the previous result for autosomal $(R=1)$ LMC models [see Eq. (3)]. 


\section{INTERPRETATION OF BIASED SEX Ratios}

The major causal theories of biased sex ratio in the scenarios discussed are within-sex competition among genetic relatives (Hamilton, 1967; Alexander and Sherman, 1977; Clark, 1978; Bulmer and Taylor, 1980a; Werren, 1980; Taylor, 1981; Charlesworth and Toro, 1982; Uyenoyama and Bengtsson, 1982), group selection (Hamilton, 1975, 1979; Colwell, 1981; Wilson and Colwell, 1981; reviewed in Charnov, 1982), and inbreeding (Maynard Smith, 1978; Stenseth, 1978; Borgia, 1982; Colwell, 1982; Uyenoyama and Bengtsson, 1982). Claims for the various theories have often been strongly worded and hotly debated. The analysis presented in this paper sheds some light on the controversy [see Nunney (1985) for a slightly different interpretation]. First, the role of inbredness in asymmetric relatedness, measured by $R$, has been clarified [see the discussion in the first scenario, LMC, and also in Uyenoyama and Bengtsson $(1981,1982)]$. But the previous literature has focused almost entirely on autosomal diploid models, where $R=1$, and so I confine my discussion to the autosomal case.

Consider the result from the LMC, LRC, and dispersal scenario; the ESS ratio males : females is $1-m\left(1-P_{d t}\right): 1-f\left(1-P_{d t}\right) . P_{d t}$ is the genetic differentiation among groups (demes), where each group has been constructed (by assumption) to be the progeny of $N$ females. It is well known that one minus the genetic differentiation among groups is the genetic correlation within groups (Wright, 1969). So, let $\rho=1-P_{d t}(0 \leqslant \rho \leqslant 1)$, where $\rho$ is the correlation of genotypic values within groups. Then the sex ratio is $1-m \rho: 1-f \rho$, where $m \rho$ is the genotypic correlation among competing males, and $f \rho$ the genotypic correlation among competing females-in other words, the levels of within-sex competition among relatives. Also note that for this scenario there is, by assumption, no inbreeding. Exactly how to measure $\rho$ is not yet known. However, we do know from other studies that when mothers control the sex ratio of their broods, settling of foundresses is random, and each foundress produces the same brood size, then $\rho=1 / N$, which is the frequency of sib competition and sib mating in these scenarios (e.g., Uyenoyama and Bengtsson, 1982).

One possibility for the meaning of $\rho$ is the regression coefficient of relatedness of controlling genotypes on competing individuals. For maternal control in a diploid organism, this regression may be taken as mothers on male progeny in the group, or on female progeny in the group, depending on the context; or, equivalently, the regression of mothers on mated composites (foundresses plus the genes of their mates). This regression for diploids is $4 F /(1+3 F)$, so the sex ratio can be written as $1+3 F-m(4 F): 1+3 F-f(4 F)$; or, the genetic value of a son is the number of genes identical by descent (ibd) from the mother minus the number 
of genes ibd (from the mother) that a son interferes with when competing for mates, and the genetic value of a daughter is the number of ibd genes in the daughter minus the number of ibd genes that a daughter interferes with while competing for a fixed limited resource.

The last scenario, LMC and dispersal, is a generalization of Hamilton's (1967) standard LMC scenario. The ESS is $1-\left(1-P_{d t}\right): 1+m\left(1-P_{d t}\right)$, or $1-\rho: 1+m \rho$, where $m$ is the proportion of males that stay with their natal deme to compete for mates. With $m=1$ the scenario is the standard LMC scenario, and the ESS is $1-\rho: 1+\rho=(1-\rho) / 2=(1 / 2) P_{d t}$. [Uyenoyama and Bengtsson (1982) report an ESS of $1-k: 1+k$, where $k$ is the proportion of sib mating, and thus also the proportion of competitive interactions among brothers. For their scenario, $k=\rho$, since they confined themselves to the case in which cofoundresses are unrelated.] In this scenario the level of inbreeding is $m \rho$, and there is no limiting resources for females, and hence no LRC. The value of a female is the Fisherian one plus the amount of inbreeding, $m \rho$. To understand how this inbreeding effect comes about, examine the expression for individual fitness, $w_{d i}$, for this scenario [Eq. (35)]. The expected number of inseminations for a male staying at home is $\left(1-r_{d}\right) / r_{d}$, while for a dispersing male, $(1-r) / r_{d}$. So making fewer males-lowering $r_{d}$-increases the value per male that stays at home. Generalizing beyond the artificial structure of the models, the genetic correlation between mates measures the relative advantage of producing a daughter rather than a son, since producing one daughter will pass on one set of parental genes, plus a second set with a probability equal to the correlation between mates, while producing one son will only pass on one set of parental genes (Fisher, 1941; Charlesworth and Charlesworth, 1978, 1981; Taylor 1981). Equation (35) is a formal statement of this phenomenon, since the index $d$ in $\left(1-r_{d}\right) / r_{d}$ refers only to the genetic composition of the set of mates available to a male, with $d=0,1,2, \ldots, N$ representing the number of $b$ types, and $q_{d}=d / N$. This inbreeding bonus for the value of a daughter still assumes the asymmetry between the sexes stated earlier: that females make babies and males just fertilize-and that each male can potentially fertilize many females. Models in which these assumptions are violated are easily constructed by modifying the expressions for $w_{d i}$. [See Uyenoyama (1984b) for a general discussion of coefficients of relatedness and group selection under inbreeding.]

Interpreting these results for the Hamilton scenario under maternal control, the value of a son is one minus the regression of mothers on male progeny in the group, while the value of a daughter is one plus the regression of mothers on daugthers' mates. Again, these regressions are equivalent to the regression of mothers on mated composites, $4 F /(1+3 F)$, so the sex ratio (with $m=1$ ) is $1+3 F-4 F: 1+3 F+4 F$, or $r^{*}=$ $(1 / 2)[(1-F) /(1+3 F)]$. Verbally, a son is worth the number of ibd genes he 
carries minus the number he interferes with, and a daughter is worth the number of ibd genes she carries plus the number of ibd genes from her mates that she transmits.

Consider an illustration of the simplicity and generality of this $\rho$ formulation. Let there be two foundresses per deme, each producing the same number of offspring, and let the expected genotypic regression between each foundress and the other mated composite be $1 / 2$. One-half of the competitive interactions, and one-half of the matings, will be among sibs $(\rho=1)$, and one-half will be among offspring of the two foundresses $(\rho=1 / 2)$, so $\rho=(1 / 2)(1)+(1 / 2)(1 / 2)=3 / 4$. For the standard LMC scenario, in which there is both inbreeding and competition among males for mates, $r^{*}=1-\rho: 1+\rho=(1 / 4):(7 / 4)=1 / 8$. For the scenario in which, by assumption, there is no inbreeding and no competition among female relatives ( $m=1$ and $f=0$ from the LMC, LRC, and dispersal scenario), $r^{*}=1-\rho: 1=(1 / 4): 1=1 / 5$. By taking care to insure that the proper meaning is given to $\rho$, it appears that this formulation allows the ESS sex ratio to be written down directly for many complex situations.

One confusion which may arise with the use of $\rho$ is the way in which the regressions are calculated. For example, consider the case in which each deme has two foundresses, one an $a$ type and the other a $b$ type. Then there is no differentiation among groups, $P_{d t}=1, \rho=0$, and biased sex ratios are not favored over the Fisherian $1 / 2$. When calculating $\rho$ for the group in this case, the proper view is that the regression of each foundress on herself is one, and of each foundress on her cofoundress, negative one, since the cofoundress is of the opposite type and equally frequent in each deme and in the population. Therefore $\rho$ is zero. When considering within-sex competition among male progeny in the deme, sib competition is associated with $\rho=1$, since sibs have the same mother, and interactions between nonsibs are associated with $\rho=-1$, for the reasons just described, so overall $\rho=0$. Hamilton (1972) has discussed the importance of negative correlations in small groups for the proper application of coefficients of relatedness.

Some have argued that since the Fisherian $1 / 2$ can be favored in a situation in which there is both sib mating and sib competition, that LMC and inbreeding alone cannot explain the evolution of biased sex ratios, and one must invoke group selection (Colwell, 1981, 1982; Wilson and Colwell, 1981). An alternative description would be that one may obtain a value of zero for $\rho$ even when there is sib mating and sib competition. Proper interpretations of within-sex competition and inbreeding depend on proper calculations of the coefficients of relatedness, and therefore the group selection approach is not different from correctly analyzed within-sex competition and inbreeding models. Colwell (1981) and Wilson and Colwell (1981) were the first to stress explicitly the correct interpretation of pop- 
ulation structure, following the cryptographic message of Hamilton (1979), and the controversy that followed focused mainly on the relationship between $\rho$ and $P_{d t}$, which should now be clear.

In summary, all three theories-inbreeding, within-sex competition among relatives, and group selection-truly describe causal mechanisms of biased sex ratios in structured populations. Through the study of a variety of scenarios with hierarchical selection theory, I draw the following conclusions. First, inbreeding biases the sex ratio since producing a daughter that inbreeds (e.g., an automictic parthenogen) passes on twice as many parental genes as producing a son would. Second, as the amount of withinsex competition among related individuals increases, the relative genetic valuation of that sex decreases. Third, genetic differentiation among groups, $P_{d t}$, and genetic correlation within groups, $\rho$, are related descriptions for the same phenomenon. Some recent papers (Colwell, 1981; Wilson and Colwell, 1981) have stressed the group selection aspect of this phenomenon without clarifying its similarity to genetic relatedness. Using group selection for describing causal mechanisms is particularly slippery, since, as in the various scenarios presented in this paper, the differentiation among groups may refer to groups of competing males, groups of competing females, or groups that contain inbreeding pairs. While hierarchical selection theory, which is a group selection sort of analysis, has proved a powerful analytical tool, it seems that, for describing causal mechanisms, it is often useful to apply the genetic regressions considered in the discussion.

\section{ACKNOWLEDGMENTS}

W. D. Hamilton and P. E. Smouse discussed these ideas with me and made many helpful suggestions on earlier drafts of the manuscript. R. K. Colwell gave a careful and penetrating review, which greatly clarified the presentation of several difficult problems, and $\mathbf{R}$. H. Crozier pointed out some important references. This project was supported by the NIH National Research Service Awards R01-GM3259 and 1-T32-07544-07 from the National Institute of General Medical Sciences.

Note added in proof. (i) In this paper the relatedness, $\rho$, of the controlling genotype to males and females of the progeny generation has been treated as a fixed parameter. Several sex-ratio models in which particular values of $\rho$ can be calculated from assumptions about population dynamics and patterns of migration are presented in a recent paper ( $\mathbf{S}$. A. Frank, in press, Heredity). These more recent models are based on numerical results presented by M. G. Bulmer (in press, Heredity). (ii) Peter D. Taylor has independently derived results that are analogous to my genetic value methodology (based on $\rho$ ) that also use Hamilton-type regression coefficients. Taylor has also proved a general theorem that formally links exact genetic models with Price covariance models (hierarchical selection), and with inclusive fitness models based on regression coeflicients. Taylor's results are contained in an unpublished manuscript, "Three models: Exact genetic, covariance and inclusive fitness." 


\section{REFERENCES}

AleXANder, R. D., AND Borgia, G. 1978. Group selection, altruism, and the levels of organization of life, Annu. Rev. Ecol. Syst. 9, 449-474.

Alexander, R. D., and Sherman, P. W. 1977. Local matc competition and parental investment in social insects, Science (Washington, D.C.) 196, 494-500.

ARnold, A. J., AND FisTruP K., 1982. The theory of evolution by natural selection: A hierarchical expansion, Paleobiology 8, 113-129.

Bateson, W., ANd Gairdner, E. 1921. Male-sterility in flax, J. Genet. 11, 269-275.

Borgia, G. 1982. Letter to Nature: Female-biased sex ratios, Nature (London) 298, 494-495.

Bulmer, M. G., AND TAYLOR, P. D. 1980a. Dispersal and the sex ratio, Nature (London) 284, 448-449.

Bulmer, M. G., AND TAYloR, P. D. 1980b. Sex ratio under the haystack model, $J$. Theor. Biol. 86, 83-89.

Charlesworth, D., and Charlesworth, B. 1978. Population genetics of partial malesterility and the evolution of monoecy and dioecy, Heredity 41, 137-153.

Charlesworth, D., and Charlesworth, B. 1981. Allocation of resources to male and female function in hermaphrodites, Biol. J. Linn. Soc. 15, 57-74.

Charlesworth, B., and Toro, M. A. 1982. Letter to Nature: Female-biased sex ratios, Nature (London) 298, 494.

Charnov, E. L. 1978. Sex ratio selection in eusocial Hymenoptera, Amer. Nat. 112, 317-326.

Charnov, E. L. 1982. "The Theory of Sex Allocation," Princeton Univ. Press, Princeton, N.J.

Charnov, E. L., Los-den Hartogh, R. L., Jones, W. T., and van den Assem, J. 1981. Sex ratio evolution in a variable environment, Nature (London) 289, 27-33.

Clark, A. B. 1978. Sex ratio and local resource competition in a prosiminian primate, Science (Washington, D.C.) 201, 163-165.

Clutton-Brock, T. H., Albon, S. D., and Guinness, F. E. 1981. Parental investment in male and female offspring in polygynous animals, Nature (London) 289, 487-489.

Colwell, R. K. 1981. Group selection is implicated in the evolution of female-biased sex ratios, Nature (London) 290, 401-404.

Colwell, R. K. 1982. Letter to Nature: Female-biased sex ratios, Nature (London) 298, 495-496.

Cosmides, L. M. AND TOOBY, J. 1981. Cytoplasmic inheritance and intragenomic conflict, $J$. Theor. Biol. 89, 83-129.

Crow, J. F., AND Kimura, M. 1970. "An Introduction to Population Genetics Theory," Burgess, Minneapolis, Minn.

Doolittle, W. F. 1982. Selfish DNA after fourteen months, in "Genome Evolution" (G. A. Dover and R. B. Flavell, Eds.), pp. 3-28, Academic Press, New York.

Doolittle, W. F., Kirkwood, T. B. L., ANd Dempster, M. A. H. 1984. Selfish DNAs with self-restraint, Nature (London) 307, 501-502.

EBERHARD, W. G. 1980. Evolutionary consequences of intracellar organelle competition, $Q$. Rev. Biol. 55, 231-249.

FISHER, R. A., 1930. "The Genetical Theory of Natural Selection," Oxford Univ. Press (Clarendon), London/New York.

Fisher, R. A. 1941. Average excess and average effect of a gene substitution, Ann. Eugen. 11, 53-63.

FISHER, R. A. 1958. "The Genetical Theory of Natural Selection," 2nd ed., Dover, New York.

FRANK, S. A. 1983a. A hierarchical view of sex-ratio patterns, Fl. Entomol. 66, 42-75.

Frank, S. A. 1983b. "Theoretical and Empirical Studies of Sex Ratios, Mainly in Fig Wasps," M. S. thesis, University of Florida, Gainesville. 
Frank, S. A. (1985). Hierachical selection theory and sex ratios. II. On applying the theory, and a test with fig wasps, Evolution.

Grafen, A. 1984. Natural selection, kin selection and group selection, in "Behavioural Ecology" (J. R. Krebs and N. B. Davies, Eds.), pp. 62-86, Sinauer, Sunderland, Mass.

Haldane, J. B. S. 1932. "The Causes of Evolution," Longmans, Green, London.

Hamilton, W. D. 1964. The genetical theory of social behaviour. I and II, I. Theor. Biol. 7, $1-16,17-52$.

Hamilton, W. D. 1967. Extraordinary sex ratios, Science (Washington, D.C.) 156, 477-488.

Hamilton, W. D. 1970. Selfish and spiteful behaviour in an evolutionary model, Nature (London) 228, 1218-1220.

Hamlton, W. D. 1972. Altruism and related phenomena, mainly in social insects, Annu. Rev. Ecol. Syst. 3, 193-232.

Hamilton, W. D. 1975. Innate social aptitudes of man: An approach from evolutionary genetics, in "Biosocial Anthropology" (R. Fox, Ed.), pp. 133-155, Wiley, New York.

Hamilton, W. D. 1978. Evolution and diversity under bark, in "Diversity of Insects Faunas" (L. A. Mound and N. Waloff, Eds.), pp. 154-175, Blackwell, Oxford.

Hamilton, W. D. 1979. Wingless and fighting males in fig wasps and other insects, in "Sexual Selection and Reproductive Competition in Insects" (M. S. Blum and N. A. Blum, Eds.), pp. 167-220, Academic Press, New York.

Hamilton, W. D., And MAY, R. M. 1977. Dispersal in a stable habitat, Nature (London) 269, 578-581.

Harvey, P. H., Partridge, L., ANd Nunney, L. 1985. Group selection and the sex ratio, Nature (London) 313, 10-11.

Herbers, J. M. 1979. The evolution of sex ratios in hymenopteran societies, Amer. Nat. 114, 818-834.

HowARD, H. W. 1942. The genetics of Armadillidium vulgare Latr. II. Studies on the inheritance of monogeny and amphogeny, J. Genet. 44, 143-159.

Laughnan, J. R. and Gabay-Laughnan, S. 1983. Cytoplasmic male sterility in maize, Annu. Rev. Genet. 17, 27-48.

Lewontin, R. C. 1970. The units of selection, Annu. Rev. Ecol. Syst. 1, 1-18.

LI, C. C. 1967a. Fundamental theorem of natural selection, Nature (London) 214, 505-506.

LI, C. C. 1967b. Genetic equilibrium under selection, Biometrics 23, 397-484.

LI, C. C. 1976. "A first Course in Population Genetics," Boxwood, Pacific Grove, Calif.

MACARThur, R. H. 1965. Ecological consequences of natural selection, in "Theoretical and Mathematical Biology" (T. H. Waterman and H. Morowitz, Eds.), pp. 388-397, Blaisdell, New York.

MacNaIR, M. R. 1978. An ESS for the sex ratio in animals, with particular reference to the social Hymenoptera, J. Theor. Biol. 70, 449-459.

MaYnard Smith, J. 1978. "The Evolution of Sex," Cambridge Univ. Press, Cambridge, Mass.

MAYNARD SMITH, J. 1982. "Evolution and the Theory of Games," Cambridge Univ. Press, Cambridge, Mass.

Maynard Smith, J. 1983. Models of evolution, Proc. R. Soc. London Ser. B. 219, 315-325.

Maynard Smith, J., AND Price, G. R. 1973. The logic of animal conflict, Nature (London) 246, 15-18.

NuNNEY, L. 1985. Female-biased sex ratios: Individual or group selection? Evolution 39, 349-361.

OHTA, K. (1983). Hierarchical theory of selection: The covariance formula of selection and its applications, Bull. Biometr. Soc. Japan No. 4, 25-33.

Pamilo, P. 1982. Genetic evolution of sex ratios in eusocial Hymenoptera: Allele frequency simulations, Amer. Nat. 119, 638-656.

Pamilo, P., AND CRozIER, R. H. 1982. Measuring genetic relatedness in natural populations: Methodology, Theor. Pop. Biol. 21, 171-193. 
PrICE, G. R. 1970. Selection and covariance, Nature (London) 227, 520-521.

PrICE, G. R. 1972a. Extension of covariance selection mathematics, Ann. Hum. Genet. 35, 485-490.

Price, G. R. 1972b. Fisher's 'fundamental theorem' made clear, Ann. Hum. Genet. 36, $129-140$.

RhoADfs, M. M. 1933. The cytoplasmic inheritance of male sterility in Zea mays, J. Genet. 27. 71-93.

Robertson, A. 1966. A mathematical model of the culling process in dairy cattle, Anim. Prod. 8, 95-108.

Robertson, A. 1968. The spectrum of genetic variation, in "Population Biology and Evolution" (R. C. Lewontin, Ed.), pp. 5-16, Syracuse Univ. Press, Syracuse, N.Y.

SEGER, J. 1981. Kinship and covariance, J. Theor. Biol. 91, 191-213.

SilAw, R. F. 1958. The theoretical genetics of the sex ratio, Genetics 93, 149163.

SHAw, R. F., AND Mohler, J. D. 1953. The selective advantage of the sex ratio, Amer. Nat. 87, 337-342.

SkINNER, S. W. 1982. Maternally inherited sex ratio in the parasitoid wasp Nasonia vitripennis, Science (Washington, D.C.) 215, 1133-1134.

STENSETH, N. C. 1978. Is the female biased sex ratio in wood lemming Myopus schistocolor maintained by cyclic inbreeding? Oikos 30, 83-89.

TAYLOR, P. D. 1981. Intra-sex and inter-sex sib interactions as sex ratio determinants, Nature (London) 291, 64-66.

TAYLOR, P. D., AND BUlmer, M. G. 1980. Local mate competition and the sex ratio, $J$. Theor. Biol. 86, 409-419.

TRIVERS, R. L., AND HARE, H. 1976. Haplodiploidy and the evolution of social insects, Science (Washington D.C.) 191, 249-263.

TRIVERS, R. L., AND WillaRD, D. E. 1973. Natural selection of parental ability to vary the sex ratio, Science (Washington, D.C.) 179, 90-92.

Uyenoyama, M. K. 1984a. On the evolution of parthenogenesis: A genetic representation of the cost of meiosis, Evolution 38, 87-102.

Uyenoyama, M. K. 1984b. Inbreeding and the evolution of altruism under kin selection: Effects on relatedness and group structure, Evolution 38, 778-795.

Uyenoyama, M. K., AND Bengtsson, B. O. 1979. Towards a genetic theory for the evolution of the sex ratio, Genetics $93,721-736$.

Uyenoyama, M. K., AND Bengtsson, B. O. 1981. Towards a genetic theory for the evolution of the sex ratio. II. Haplodiploid and diploid models with sibling and parental control of the brood sex ratio and brood size, Theor. Pop. Biol. 20, 57-79.

Uyenoyama, M. K., AND BENGTSSON, B. O. 1982. Towards a genetic theory for the evolution of the sex ratio. III. Parental and sibling control of brood investment ratio under partial sib-mating, Theor. Pop. Biol. 22, 43-68.

Uyenoyama, M. K., ANd Feldman, M. W. 1981. On relatedness and adaptive topography in kin selection, Theor. Pop. Biol. 19, 87-123.

Uyenoyama, M. K., and Feldman, M. W. 1982. Population genetic theory of kin selection. II. The multiplicative model, Amer. Nat. 120, 614-627.

WADE, M. J. 1980. Kin selection: Its components, Science (Washington, D.C.) 210, 665-667.

WADE, M. J. 1985. Soft selection, hard selection, kin selection, and group selection, Amer. Nat. $125,61-73$.

WARD, P. S. 1983. Genetic relatedness and colony organization in a species complex of ponerine ants. II. Patterns of sex ratio investment, Behav. Ecol. Sociobiol. 12, 301-308.

WerREN, J. H. 1980. Sex ratio adaptations to local mate competition in a parasitic wasp, Science (Washington, D.C.) 208, 1157-1159.

WERREN, J. H. 1983. Sex ratio evolution under local mate competition in a parasitic wasp, Evolution 37, 116-124. 
Werren, J. H., and Charnov, E. L. 1978. Facultative sex ratios and population dynamics, Nature (London) 272, 349-350.

Werren, J. H., Skinner, S. W., and Charnov, E. L. 1981. Paternal inheritance of a daughterless sex ratio factor, Nature (London) 293, 467-468.

WiLDISH, D. J. 1982. Letter to Nature: Female-biased sex ratios, Nature (London) 298, 495.

WILson, D. S. 1980. "The Natural Selection of Populations and Communities," Benjamin/Cummings, Menlo Park, Calif.

WILSON, D. S. 1983. The group selection controversy: History and current status, Annu. Rev. Ecol. Syst. 14, 159-187.

WILSON, D. S., AND COLWELl, R. K. 1981. Evolution of sex ratio in structured demes, Evolution 35, 882-897.

WRIGHT, S. 1969. "Evolution and the Genetics of Populations," Vol. 2, Univ. of Chicago Press, Chicago.

Zimmering, S., Sanderr, L., ANd Nicoletti, B. 1970. Mechanisms of meiotic drive, Annu. Rev. Genet. 4, 409-436. 